\title{
Strates
}

STRATES Matériaux pour la recherche en sciences sociales

$11 \mid 2004$

Jeune recherche, la vitalité d'un laboratoire

\section{Les déchets ménagers : pour une recherche interdisciplinaire}

Emmanuelle Le Dorlot

\section{(2) OpenEdition}

1 Journals

Édition électronique

URL : http://journals.openedition.org/strates/410

DOI : $10.4000 /$ strates. 410

ISSN : $1777-5442$

Éditeur

Laboratoire Ladyss

Édition imprimée

Date de publication : 1 janvier 2004

ISSN : 0768-8067

Référence électronique

Emmanuelle Le Dorlot, «Les déchets ménagers : pour une recherche interdisciplinaire ", Strates [En ligne], 11 | 2004, mis en ligne le 14 janvier 2005, consulté le 08 septembre 2020. URL : http://

journals.openedition.org/strates/410; DOI : https://doi.org/10.4000/strates.410

Ce document a été généré automatiquement le 8 septembre 2020

Tous droits réservés 


\title{
Les déchets ménagers : pour une recherche interdisciplinaire
}

\author{
Emmanuelle Le Dorlot
}

État des lieux : un quasi vide en sciences sociales

1 Marginal dans la société, et même dans la sphère politique puisque la législation en la matière est très récente $(1975,1992)$, les déchets ménagers l'ont été aussi dans la recherche scientifique ${ }^{1}$, bien qu'ils représentent des problèmes et des risques de pollution évidents. Ceci est particulièrement vrai dans les sciences sociales qui ont peu investi ce domaine alors que leur place y était légitime. Les recherches menées sont principalement techniques et placent la solution technique en leur centre. L'examen de la production bibliographique de l'Ademe et du ministère de l'Environnement, comme leurs appels d'offre de recherche, en apporte une confirmation et révèle le peu de confiance faite à la recherche autre que technique sur cet objet.

2 L'image sociale du déchet, ambiguë, peut expliquer l'origine de ce quasi vide en sciences sociales. Le déchet est à la fois le banal, l'inutile, mais il est aussi ce qu'on rejette, voire le répulsif. Comme l'écrit Jean Gouhier : « Le terme exprime d'abord une dépréciation réelle et constatée : le bien n'a plus de fonction affectée et par conséquent plus d'ancrage géographique. En outre, on ajoute des torts à son inutilité, il est gênant parce qu'il encombre et parce qu'il témoigne d'un refus. Il faut donc l'éloigner, le mettre en marge. Enfin pour mieux fonder son exclusion, on lui attribue une nuisance plus ou moins fondée: laid, sale, malsain, dangereux, il paraît nécessaire de le dissimuler, de l'enfouir, de le détruire ${ }^{2} »$.

3 Il s'est donc construit un «décalage » entre la représentation sociale du déchet et la gravité du problème environnemental qu'il pose, avec sans doute, pour conséquence, le faible investissement scientifique dans ce domaine. L'objectif que nous poursuivons est de montrer qu'il s'agit d'une question aussi importante à instruire que l'effet de serre, la perte de biodiversité et qui devrait être un des programmes de recherche central sur le développement durable.

4 Un état de l'art des publications en sciences humaines constitue un préambule nécessaire. 
Du côté de la géographie

Qu'il s'agisse de la géographie physique ou de la géographie sociale, les déchets ménagers n'ont jamais été un objet central de la discipline. Les deux premiers chercheurs géographes, Jean Gouhier et Albert Tauveron, à être des spécialistes et des pionniers dans leur discipline sur ce sujet ont eu du mal à être reconnus. Leurs recherches, bien que novatrices, ont été peu considérées car elles n'entraient pas dans les paradigmes, les débats théoriques et les problématiques légitimes de la géographie.

Albert Tauveron a une vision politique, économique et technique du sujet. Dans l'un de ses ouvrages, Les années poubelle (1984), il fait le tour de la question du gaspillage, en analysant les différentes méthodes de traitement existantes, en expliquant les rouages du système de production-distribution, la faiblesse de la réglementation. Il propose déjà aux débuts des années 80 des idées qui resurgissent aujourd'hui : la question des normes des produits, celle des taxes sur les produits jetables. Albert Tauveron a montré l'enjeu et les problèmes de la gestion des déchets d'une manière assez novatrice pour l'époque.

7 Les recherches de Jean Gouhier portent sur l'analyse de l'interface entre ce que l'on pourrait appeler les systèmes sociaux (population, origine ou niveau social, comportement) et les déchets (nature, production), et les implications de cette interface dans l'espace. Une citation revient souvent lorsque l'on évoque ses recherches: «Montre-moi ta poubelle, je te dirais qui tu es!» Il aborde aussi la question de la marginalité du déchet et de la marginalité des espaces où ils se situent. Les marges et les espaces marginaux sont un écho à la marginalité du déchet. À une image négative répond un espace sans usage et sans valeur. Fort de l'étendue de ses recherches dans ce domaine, il a même créé la science des déchets : la rudologie ${ }^{3}$ en 1985 et un Institut de rudologie (association régie par la loi de 1901) associé par convention à l'université du Maine et soutenu par la région des Pays de la Loire. Les relations fortes avec des partenaires politiques et économiques fait, de cet Institut, un centre de recherche à la fois fondamentale et appliquée. C'est ce que Jean Gouhier nomme la recherche action. Cet Institut délivre un ensemble de formation destinées à la fois aux étudiants et aux professionnels, agents de la sphère économique et politique. Il s'est formé une véritable école au sein de l'université du Maine (Le Mans) au travers du DESS de rudologie dirigé par Jean-René Bertrand, successeur de Jean Gouhier et de l'équipe de recherche Gregum du laboratoire ESO. Parmi les dernières thèses, on peut citer celle d'Urbain Anselme N'Kounkou qui a pour titre «Gestion territoriale des ordures ménagères franciliennes - le gisement des ordures ménagères, leurs espaces de production : vers une minimisation du flux résiduel » (2000). Cette thèse démontre qu'il existe une influence du lieu d'habitation et du type d'habitat sur les habitudes de consommation des habitants et par conséquent sur le gisement potentiel des ordures ménagères d'un point de vue qualitatif. L'hypothèse qui est avancée est que les objectifs de réduction de la production des ordures et des dysfonctionnements liés à leurs flux ne peuvent être atteints que par un système de mesure des rejets des ménages. Cette recherche a utilisé l'outil construit par l'Ademe (Modecom), outil de caractérisation des ordures ménagères. On imagine très bien les applications politiques de ces résultats notamment la construction de données pour mettre en place des collectes sélectives. En dépit de leur production scientifique, des liens qu'ils établissent avec les acteurs impliqués dans ce domaine, malgré un début de reconnaissance par les collectivités locales de l'intérêt des approches de ce problème par la géographie sociale, les 
géographes travaillant sur ce domaine souffrent d'isolement dans leur discipline. Il existe une force de recherche insuffisante sur ce thème.

Du côté des autres sciences humaines

8 Les déchets ménagers semblent avoir suscité plus d'intérêt chez les sociologues et les économistes.

9 L'approche économique peut se diviser en deux axes majeurs. Le premier s'interroge sur l'efficacité économique d'un service public local et des différentes techniques de collecte et de traitement par rapport au marché économique (Defeuilley, 1996). Il est question des coûts, d'interactions entre la gestion, le financement et la tarification (Le Bozec, 1994). Le Bozec se distingue de Defeuilley en introduisant les concepts d'intercommunalité et en accordant une place importante aux acteurs.

Un deuxième axe, plus global et plus industriel, pose les questions de cycle du produit, de sa durée de vie, du recyclage, de la récupération, du gaspillage, revers de la médaille du système économique (Bertolini, 1978-1996). D'autres s'intéressent plus spécifiquement à une technique. C'est le cas de Courtine (1996) par rapport à la décharge ou de Le Goux et Le Douce par rapport à l'incinération (1995). Il semble que l'économie ait trouvé une plus grande légitimité par rapport à la géographie. Ceci est dans doute dû aux enjeux économiques et même financiers qui se sont créés autour des déchets, véritable objet marchand. C'est bien sûr, un point de vue dont il faut tenir compte.

11 L'approche sociologique met en avant les représentations des déchets et les comportements des acteurs. C'est la question du tri et de la collecte sélective (compréhension du geste du tri, des facteurs d'influence des comportements face aux déchets et au tri) qui a mobilisé la plupart des recherches dans cette discipline. On peut citer les travaux sociologiques et/ou socio-économiques de Bruno Maresca et Guy Poquet du Credoc (1994), de Laredo et Barbier (1999), de Charvolin (1998-1999), Goaquin (1994). Bruno Maresca et Guy Poquet s'intéressent aux comportements spécifiques du tri. Sont abordés successivement l'évolution de la collecte sélective au travers des réglementations et des expériences de terrain (District de l'agglomération nancéienne constituée de 18 communes, le Syndicat départemental pour les ordures ménagères (Sydom) du Jura et la ville des Mureaux dans les Yvelines) et surtout les principes du tri (quels sont les facteurs de participation et de mobilisation au geste du tri ?). Laredo et Barbier se sont intéressés au concept d'internalisation à travers l'étude de cas de la Communauté Urbaine de Lille (CUL). Par internalisation, les auteurs entendent l'intégration, notamment en matière de coût, des avantages et des contraintes liés à l'activité autour des déchets ménagers et aux multiples relations entre les acteurs afin de réguler le marché des déchets. Sont abordés la problématique de l'internalisation, le processus de modernisation de la politique de la CUL et les problèmes que celui-ci a rencontrés, et enfin la question de savoir comment transformer l'usager en producteur-trieur et banaliser de nouvelles pratiques de tri. Rémy Barbier dans sa thèse de doctorat (1996) analyse le tri et propose une réinterprétation du phénomène Nimby dans une perspective non critique permettant de caractériser de nouvelles modalités de formation et d'expression d'un jugement public sur les techniques.

12 L'approche sociologique a sa valeur, mais, pour que la question des déchets deviennent véritablement une question scientifique à portée finalisée, il serait nécessaire de l'articuler à l'approche géographique et de la référer au problème environnemental dans sa complexité. En effet, contrairement à la géographie, la sociologie semble 
accorder peu d'intérêt d'une part à la relation, pourtant essentielle, des sociétés à leurs lieux et milieux et d'autre part à la matérialité, à la réalité physique du déchet qui conditionne fortement sa gestion. Les déchets ménagers sont un objet complexe et mixte pour reprendre les termes employées par Nicole Mathieu et Marie Claire Robic sur le développement durable ${ }^{4}$, "c'est-à-dire un objet dans lequel les dimensions naturalistes (matérielles) et les dimensions sociales (représentations-pratiques, conflits-pouvoirs, appropriation-exclusion) ne peuvent être dissociés ».

Une réelle complémentarité existe entre la géographie et la sociologie qu'il faudrait mettre en œuvre. Les déchets ménagers par ses multiples dimensions sont un objet porteur d'une mise en commun de concepts et méthodes permettant de le comprendre, voire d'apporter des éléments de réponses à l'apparente insolvabilité de ce problème d'environnement.

De la maîtrise à la thèse : acquis des recherches antérieures

14 Face à un champ d'étude vaste et à la variété des approches de la question, nos premières recherches se sont faites sur des choix. Ils sont d'abord thématiques: évaluation des politiques publiques de gestion des déchets ménagers à deux échelles (nationale à travers la législation et locale à travers les moyens mis en œuvre sur le terrain des collectivités locales des Hautes-Pyrénées pour gérer les déchets) et méthodologiques : priorité à l'exploration de terrain et à une méthode inductive fondée sur une imprégnation dans ces terrains et la construction d'informations pertinentes pour la question.

15 Sur un sujet par essence pluridisciplinaire, même si la géographie est au cœur de notre recherche tant par les références méthodologiques que les concepts mobilisés, l'emprunt à la sociologie et à la science politique est nécessaire. Il a fallu aussi tenir compte des connaissances techniques dans la matière. L'approche de terrain et l'analyse locale nous a permis de tendre vers cet objectif pluridisciplinaire.

16 Pour être au cœur du débat, le chercheur ne doit pas avoir peur de «vivre sa recherche » d'où la nécessité de s'imprégner, de baigner dans le décor qu'il analyse. Les enquêtes que nous avons faites dans le département des Hautes-Pyrénées y ont contribué, mais les nombreuses visites d'équipements de traitement des déchets ménagers, décharges, UIOM, déchetteries... plus encore. Il est difficile de comprendre le phénomène sans avoir vu de ses propres yeux une décharge, avoir marché sur le dépôt, s'être rendue compte des dizaines de mètres sous ses pieds, sans s'être placée devant la fosse d'accueil des déchets d'une UIOM, sans avoir été assise dans le fauteuil du technicien qui manipule le grappin, qui amène les déchets de la fosse au four d'incinération, sans avoir ne serait-ce qu'entr'aperçu les flammes brûlant des milliers de tonnes de déchets à près de $800^{\circ} \mathrm{C}$. Toutes ces expériences, gravées dans la mémoire, permettent de comprendre le fonctionnement de ce secteur et l'urgence de faire évoluer les choses.

17 Ce regard géographique et global sur le secteur des déchets au travers d'une étude locale de six ans sur le département des Hautes-Pyrénées ${ }^{5}$ nous a permis de démontrer et de mettre en évidence la dimension locale de la gestion des déchets ménagers, révélant une réalité qui contraste très nettement avec l'idéal de gestion énoncé dans la loi. Sur le terrain, les élus, qu'ils appartiennent à des syndicats intercommunaux ou non, sont dépassés par le problème des déchets ménagers de plus en plus abondants. Ils font difficilement face et sont aux limites de leurs compétences techniques en matière de traitement d'autant que ces limites ne font que croître avec la parution de normes 
environnementales de plus en plus sévères et de techniques de traitement de plus en plus sophistiquées. Cette solitude de l'élu local (surtout ceux des communes rurales) face à un problème environnemental complexe aboutit à des incohérences politiques, à des pollutions qui ne font qu'accentuer une situation environnementale déjà critique. D'autre part, les techniques en usage sur notre terrain en matière de traitement ont montré leurs limites en tant que solution aux problèmes environnementaux posés par les déchets ménagers. L'analyse historique des techniques de traitement employées sur les décharges des Hautes-Pyrénées a démontré que l'apport successif de nouvelles techniques et méthodes de traitement ne faisait que déplacer le problème sans pour autant le résoudre. Cette gestion technicienne des déchets produit une fracture entre les objectifs de l'Etat et leur application au niveau local par les collectivités locales.

Notre recherche a, par ailleurs, mis en évidence l'origine et les diverses contraintes qui expliquent ce décalage. La place réservée à la technique comme solution unique au problème des déchets en est une. L'absence de prise en compte du coût d'une politique telle qu'elle a été définie par l'Etat en est une autre. Protéger l'environnement et gérer de manière écologique les déchets ménagers ont un coût élevé à court terme, un coût mal perçu et mal compris car le long terme n'est pas ancré dans les politiques, les comportements et la culture de tout un chacun. Nous dirons que la question du coût est secondaire car elle dépend d'une réelle volonté politique, celle de consacrer un budget à un problème.

19 Les contraintes majeures sont occultées dans la politique de l'Etat et celles des collectivités locales du fait de l'absence d'une territorialisation de la gestion des déchets, d'un ancrage local et de la prise en compte de l'espace dans ses caractéristiques physiques, humaines, architecturales... La primauté de la technique n'est pas sans relation avec cette non-spatialisation et ce non-ancrage territorial. Cette position politique a pour conséquence le fait que chaque élu copie la politique de l'autre alors qu'elle n'est pas forcément adaptée à son territoire. D'où une incohérence des politiques, l'augmentation des pollutions partout, et les échecs en matière de gestion des déchets ménagers... Enfin, la dernière contrainte majeure, contrainte ignorée des stratégies de gestion des déchets ménagers, est celle des comportements humains. De nombreux comportements politiques, ou simplement anti-citoyens, sont en effet à l'origine de la faible évolution sur le terrain de la politique des déchets ménagers. Dans le monde politique, c'est la vision à court terme, les stratégies électorales et les batailles de clocher qui sont à l'origine des inerties locales. Du côté des individus, le comportement Nimbyiste (Not in my back yard: " pas de ça chez moi », opposition intempestive et pas toujours objective aux projets en matière de gestion des déchets ménagers), l'individualisme en contradiction avec la nécessité d'une responsabilité collective, le poids des habitudes et enfin le conservatisme lié à une absence de vision projective du problème des déchets ménagers au-delà du service journalier, sembleraient les causes majeures de l'inertie.

20 Les réponses à ces contraintes sont donc, au-delà de la nécessité de l'ancrage au niveau local de toute politique ${ }^{6}$, le dépassement des comportements individualistes, qu'ils soient ceux des personnalités politiques ou des citoyens ordinaires. Pour arriver à ces dépassements, il est nécessaire d'intégrer dans les politiques locales de gestion des déchets ménagers, de l'information, de la sensibilisation, de la concertation et de la responsabilité. Ce n'est que par une réelle connaissance du phénomène déchets et de l'action des élus que les individus pourront durablement changer les comportements, 
trier leurs déchets, ne plus jeter n'importe comment et n'importe où leurs déchets. Enfin, il est nécessaire que la gestion individuelle devienne collective. Elle doit réunir l'ensemble des acteurs, aussi diverses soient leurs stratégies, pour établir cette chaîne qui va de l'individu à l'industriel producteur de biens de consommation.

21 Cette recherche a eu quatre intérêts majeurs: mise en évidence d'une situation complexe et problématique, identification de ses causes, proposition de solutions originales et construction d'un regard géographique sur la question. Le développement d'une recherche scientifique comme la nôtre dans ce domaine a montré qu'il est nécessaire que les sciences humaines, en particulier, se confrontent à une réalité concrète, gérée par des acteurs dont la vision est principalement technique. Gestion collective, responsabilité et vision à long terme doivent s'allier à l'invention et à l'utilisation de procédés industriels. C'est aux sciences humaines en particulier de le démontrer grâce à leur point de vue particulier, novateur dans ce domaine très technicisé. L'indépendance de la recherche dans ce type d'étude est primordiale pour élargir et renouveler cette question. Par nos résultats, nous pensons avoir contribué à faire admettre que les sciences sociales, la géographie en particulier, ont un rôle à jouer pour que la question des déchets ménagers prenne une place à part entière dans les problématiques environnementales.

Depuis la thèse, nos travaux ont du se limiter à une veille scientifique sur ce problème d'environnement. Ils se sont traduits par des contributions dans la revue Natures Sciences Sociétés qui en reconnaît l'importance, la nécessité de le traiter comme un problème interdisciplinaire et donc par des disciplines des sciences sociales.

Aujourd'hui, notre position s'est consolidée et peut s'exprimer dans les termes suivants. Insuffisamment étudié par les géographes, le déchet est un objet géographique au sens propre dans la mesure où la question qu'il pose est au cœur d'un rapport triangulaire déchet/homme/espace. Les concepts de dispersion/accumulation, isolement, distance/proximité sont des enjeux importants voire centraux dans la problématique actuelle de cet objet. La généralisation des modes de consommation qui sont ceux de la société de consommation (augmentation des produits, des suremballages...) et l'étalement de l'urbanisation ont modifié les systèmes de régulation qui étaient fondés sur la dispersion des points de production. Par un ensemble de réactions en chaîne, cela a conduit à faire des déchets ménagers, un problème environnemental majeur. L'accumulation des déchets a accru la pression environnementale dans l'espace de leur traitement, pression d'autant plus forte que la décharge et le simple concept d'élimination ont été le fil conducteur des politiques locales de gestion des déchets ménagers. C'est également l'accumulation dans des espaces réduits des tonnes de déchets qui a rendu les risques des déchets plus importants. Malgré l'ensemble de ces risques potentiels de nuisances, le rapport de force entre déchet/espace a été nié car le concept d'élimination a prédominé. La décharge et l'incinération n'étant que les exutoires physiques et les manifestations techniques de celui-ci.

24 L'éloignement a été le maître mot du rapport homme/déchet. Parce que le déchet est une nuisance, un rejet, il est nécessaire de s'en éloigner, de l'éliminer (éliminer, du latin eliminare: mettre dehors, de limen: seuil). Le seul destin du déchet est d'être évacué le plus loin possible de son lieu de production, d'être caché au regard de ceux qui l'ont produit. Ainsi, le déchet, pourtant omniprésent dans notre société, échappe par la rapidité de son évacuation (poubelle, vide-ordures, bennes de collecte) vers des 
centres de traitement situés dans des lieux marginaux et périphériques, volontairement isolés du paysage qui les entourent comme pour accentuer leur isolement et la distance entre l'homme et le déchet. C'est ce que nous avons constaté dans notre espace d'étude pendant nos recherches. En effet, l'isolement est l'aspect commun le plus frappant des centres de traitement des Hautes-Pyrénées (décharges en l'occurrence). Deux facteurs géographiques et paysagers contribuent à cet isolement : le site même et sa qualité d'invisibilité. Les sites occupent souvent des vallons et se fondent dans des ensembles collinaires limitant ainsi les possibilités de visibilité de la décharge. Les angles de vue sont limités par l'aspect escarpé et le relief. L'autre facteur est l'aspect boisé des sites. Cumulé au relief, l'aspect boisé amplifie l'isolement du dépôt et sa faible visibilité dans un rayon important. Une deuxième distance se superpose à l'isolement. L'éloignement des sites par rapport à la majeure partie des lieux de production. Une réelle distance entre le lieu de traitement et le lieu de production s'est construite.

Il y a donc une relation évidente entre la distance au déchet et la perception du déchet et du risque du déchet. Plus le déchet est éloigné, inexistant, plus on le minimise lui et ses risques. Enfoui dans les profondeurs de la poubelle, du vide-ordures, de la benne, de la décharge ou de l'UIOM, le déchet n'existe plus. Une nouvelle décharge, une nouvelle usine d'incinération s'installe et c'est tout un pan d'individus qui crie à une future crise environnementale et qui s'oppose au projet. C'est ce que l'on appelle le phénomène Nimby. Le phénomène Nimby n'est autre qu'une réaction locale. Cela prouve que la distance a un lien de causalité avec l'enclenchement d'une opposition et d'une manifestation par rapport à la perception d'un ou plusieurs risques.

Lorsque l'on aborde le concept de valorisation, c'est la proximité qui est nécessaire à celle-ci. Proximité entre l'homme et le déchet par la manipulation lors du tri. Le tri induit un rapport plus étroit avec le déchet puisqu'il faut le regarder, l'analyser pour savoir en quelle matière il est, puis le déposer dans des équipements spécifiques: container, déchetterie, espace propreté, poubelle de tri. Une autre proximité doit s'installer, celle entre l'homme et les lieux de collecte sélective pour réduire les efforts des individus et faire en sorte que la distance ne soit pas une contrainte et un facteur limitant dans l'utilisation de l'équipement. Dans le domaine de la collecte sélective et en regard de l'usager, la distance est non seulement une mesure spatiale, c'est-à-dire un kilométrage, mais c'est aussi une notion temporelle (le temps à consacrer au dépôt des déchets triés c'est-à-dire entre l'habitat et l'équipement d'apport volontaire). Aussi importante que la distance physique, la distance temporelle entre deux points est un élément essentiel à l'usager. Le tri sélectif, en lui-même, amène une consommation de temps, celle-ci ne doit pas s'agrandir dans le déplacement de l'usager. La distancetemps doit être perçue comme une condition de confort d'utilisation et non d'effort.

Malgré les nombreux concepts géographiques qui sont mobilisés par la question des déchets, ceux-ci restent encore à explorer par les géographes, contribuant à un renouvellement du regard sur les déchets et participant à mieux maîtriser ce problème environnemental.

L'approche spatiale et l'analyse des interactions (interactions entre individus, interactions entre individus, activités et espaces) permettraient d'avoir un regard plus global sur le sujet et être à même de mettre en évidence certaines relations qui sont encore ignorées. L'approche géographique en partant de l'espace permettrait d'avoir une approche large et englobant tous les aspects et surtout les acteurs de ce domaine, 
de "dépasser les cloisonnements thématiques (voire des disciplines), scalaires pour retrouver les processus généraux à l'œuvre " ${ }^{7}$.

De la variabilité temporelle des déchets ménagers à l'analyse de la localisation des centres de traitements, des impacts de ces derniers sur l'espace environnant aux implications spatiales des difficultés d'application des normes et des objectifs qu'ils soient nationaux ou européens, des conséquences de l'harmonisation européenne aux politiques différenciées des pays, de l'analyse des comportements des individus à celle du phénomène plus particulier du Nimby, du rapport entre politique et déchet à la relation entre statut social du déchet et gestion des déchets, les thèmes de recherche sont légions et très abondants.

A travers l'état des lieux de la recherche ainsi que par le rappel de nos travaux et de leurs principaux résultats, nous espérons avoir démontré qu'il existe un domaine scientifique qui mérite un investissement des sciences sociales et de la géographie en particulier.

\section{BIBLIOGRAPHIE}

Barbier R., 1996, La recherche-action, Paris, Anthropos.

Bertolini G., 1978, La socio-économie du déchet, Éditions Entente, Collection Cahiers de l'écologie.

Bertolini G., 1978, Rebuts ou ressources, Entente, Les Cahiers de l'écologie.

Bertolini G., 1990, Le marché des ordures : économie et gestion des déchets ménagers, Ed. L'Harmattan, Collection Environnement. Courtine, 1996,

Bertolini G., 1991, Homo Plasticus : les plastiques, défi écologique, Sang de la Terre.

Bertolini G., 1996, Déchet, mode d'emploi, Economica.

Chalot F., 1990, La commune et les déchets, Ed. Sorman.

Charvolin F., 1998, «Le 'trieur' : essai d'une problématique sociologique relative aux usagers de la collecte sélective des ordures ménagères ", in École des Mines de Saint-Etienne. Traduction des comportements humains dans la simulation des projets de collecte et de traitement des déchets ménagers.

Defeuilley C., 1996, Le service public au défi de l'efficacité économique. Les contrats de délégation dans la gestion des déchets ménagers, Thèse de Doctorat de Sciences Economiques, Université Paris VII.

Gouhier J., 1984, Géographie des déchets : l'art d'accomoder les restes, Paris, Centre de documentation industrielle, Centre Georges Pompidou.

Guellec A., 1997, Déchets ménagers, pour un retour à la raison, rapport au Sénat.

Laredo Ph. et Barbier R., 1999, L'internalisation des déchets, Economica.

Le Bozec A., 1994, Le service d'élimination des ordures ménagères : coûts, organisation, gestion. Analyse systémique et économique du recyclage des ordures. Ed. L'Harmattan/CEMAGREF.

Le Goux J.-Y. et Le Douce C., 1995, L’incinération des déchets ménagers, Economica. 
Maresca B. et Poquet G., 1994, Collectes sélectives des déchets ménagers et comportements des ménages, Credoc, coll. de rapports.

N'Kounkou U. A., 2000, thèse Gestion territoriale des ordures ménagères franciliennes - le gisement des ordures ménagères, leurs espaces de production : vers une minimisation du flux résiduel.

Présentation de l'Institut de Rudologie, 1996, Université du Maine.

Robic M.-C. et Mathieu N., 2001, « Géographie et durabilité : redéployer une expérience et mobiliser de nouveaux savoir-faire ", Le développement durable, de l'utopie au concept. Éd. Elsevier SAS.

\section{NOTES}

1.De nombreux ouvrages traitent de la gestion des déchets ménagers en se plaçant du côté de l'élu local. C'est le cas de l'ouvrage de Francis Chalot (1990) et du rapport du député Ambroise Guellec (1997). Malgré leur intérêt informatif, ils n'ont pas de caractères scientifiques. On peut y ajouter la plupart des études de l'Ademe et de la société Eco-Emballages.

2.Jean Gouhier, Géographie des déchets : l'art d'accomoder les restes, Paris, Centre de documentation industrielle, Centre Georges Pompidou, 1984, 18 p.

3. «a rudologie est l'étude systématique des déchets, des biens et des espaces déclassés. C'est aussi l'étude du fondement et des motivations du geste du rejet et de la mise en marge. C'est enfin une lecture nouvelle de la marge économique et sociale pour en faire le chantier de nouvelles ressources et de nouvelles fonctions ». Présentation de l'Institut de Rudologie, université du Maine, 1996, 6 p.

4. Marie Claire Robic et Nicole Mathieu, « Géographie et durabilité : redéployer une expérience et mobiliser de nouveaux savoir-faire ", Le développement durable, de l'utopie au concept. Éditions scientifiques et médicales Elsevier SAS, 2001, pp. 167-190.

5.De la maîtrise à la thèse de doctorat.

6.C'est-à-dire de la prise en compte des caractéristiques spatiales globales des territoires.

7.Rapport d'activité du laboratoire Ladyss, 1996/1998.

\section{RÉSUMÉS}

Bien que posant de graves problèmes de gestion liés à leur volume croissant et aux risques de pollutions qu'ils génèrent, les déchets ménagers n'ont pas suscité et ne suscitent pas l'enthousiasme des chercheurs mobilisés par les questions environnementales en particulier dans les sciences sociales. Après l'avoir montré par un état des lieux des travaux en sociologie, économie et géographie, l'auteur tente de convaincre de la légitimité de cet objet de recherche en faisant un retour sur les travaux qu'elle a engagé pour montrer l'intérêt d'une approche géographique de ce problème de gestion. Cette démarche met en avant l'importance de la configuration matérielle et physique des territoires de la gestion ainsi que celle de la distance qui 
joue un rôle déterminant dans l'efficacité et la durabilité de la politique des déchets ménagers. Mais si le déchet, par sa complexité, est un objet géographique, le problème de sa gestion ne peut se passer de l'analyse sociologique des formes de concertation entre les acteurs qui concourent au contrôle de leur production et des risques induits et prévisibles.

Although kitchen waste creates serious problems of management because of its growing volume and the risks of pollution it generates, the issue still has not aroused the enthousiasm of the researchers concerned with environmental questions, in particular in social sciences. After demonstrating this through an assessment of the research in sociology, economy and geography, the author attempts to convince us of the legitimacy of this topic of investigation by coming back on the studies she initiated to show the relevance of a geographical approach of this problem of management. This approach highlights the importance of the material and physical configuration of the management territories as well as the importance of distance which plays a crucial role in the efficiency and durability of kitchen waste policies. But if waste, because of its complexity, is a geographical object, the question of its management is indissociable from a sociological analysis of the forms of the dialogue between the actors who take part in the control of its production and of the subsequent, foreseeable risks.

INDEX

Keywords : France

Mots-clés : Interdisciplinarité, Déchets

\section{AUTEUR}

EMMANUELLE LE DORLOT

Géographe. Doctorat, Ladyss, université de Paris 1, ledorlot@yahoo.fr 